\title{
Kinetics and Jamming Coverage in a Random Sequential Adsorption of Polymer Chains
}

\author{
Jian-Sheng Wang and Ras B. Pandeyt \\ Department of Computational Science, National University of Singapore, Singapore 119260, Republic of Singapore
}

(6 May 1996)

\begin{abstract}
Using a highly efficient Monte Carlo algorithm, we are able to study the growth of coverage in a random sequential adsorption (RSA) of self-avoiding walk (SAW) chains for up to $\sim 10^{12}$ time steps on a square lattice. For the first time, the true jamming coverage $\left(\theta_{J}\right)$ is found to decay with the chain length $(N)$ with a power-law $\theta_{J} \propto N^{-0.1}$. The growth of the coverage to its jamming limit can be described by a power-law, $\theta(t) \approx \theta_{J}-c / t^{y}$ with an effective exponent $y$ which depends on the chain length, i.e., $y \simeq 0.50$ for $N=4$ to $y \simeq 0.07$ for $N=30$ with $y \rightarrow 0$ in the asymptotic limit $N \rightarrow \infty$.
\end{abstract}

82.20.Wt, 82.20.-w, 81.05.Qk, 68.45.Da

Studying the kinetics of random sequential adsorption (RSA) has attracted a considerable interest in recent years [1 18] because of its enormous applications 1924 in the adsorption processes involving a variety of species from a point-like particle to a protein-like complex structure in physical, chemical, and biological systems. Some of the examples include binding of ligands on polymer chains, coating, designing composites, chemisorption, physisorption, and reaction of molecular species including globular protein on surfaces and interfaces, etc. These adsorption processes may be divided into two categories: (1) annealed adsorption where the species are mobile (a thermal equilibration for the interacting adsorbants) before they settle onto the surface - a cooperative sequential adsorption. (2) Quenched adsorption where the adsorption occurs without subsequent diffusion or desorption. We consider the latter category known as random sequential adsorption.

The problem of RSA in one dimension [19,25, 27] is well understood with exact results for some adsorption processes. Understanding the growth of coverage in two dimensions with the RSA lacks rigorous results by analytical methods due to their intractabilities especially for objects with polydisperse shapes. Therefore, computer simulations remain one of the primary tools to investigate these problems. Numerical results [2] and theoretical analyses 3. 13 for the deposition of disks on continuum suggest that the coverage follows the Feder's law [2] at large time,

$$
\theta(t) \approx \theta_{J}-\frac{c}{t^{1 / d}},
$$

where, $\theta(t)$ is the coverage at time $t, \theta_{J}$ is the jamming coverage, $d$ is the dimensionality of the host space, and $c$ is a constant.

The temporal dependence, Eq. (11), seems to describe the deposition of hyperspheres. For squares [7], rectangles [6], and ellipses [14], on the other hand, the above law is still valid but $d$ is replaced by $d_{f}$, the number of degrees of freedom of the corresponding objects. For disks with polydisperse sizes the computer simulation studies 13. seem consistent with the theoretical prediction [12], $\theta(t) \approx \theta_{J}-c t^{-y}$, where $y=1 /(d+1)$. As a natural extension, one would like to consider objects not only with polydisperse sizes but also with polydisperse shapes, a more complex problem in the studies of RSA. Therefore, we perform a large-scale Monte Carlo simulation to study the adsorption of polymer chains which have polydispersity in both shapes and sizes. A model chain is one of the most simple ramified objects with its well-known shape and size distributions in a variety of systems [28,29]. The RSA of polymer chains is nevertheless relevant in applications such as coating and paint. In contrast to theoretical predictions for the RSA of polydisperse objects of regular shapes, we find that an effective exponent $y$ depends on the chain length. Furthermore, we are able to reach the true jamming coverage $\left(\theta_{J}\right)$ with an efficient algorithm which enables us to predict a power-law dependence on the chain length $(N), \theta_{J} \propto N^{-0.1}$.

We consider an $L \times L$ square lattice with a periodic boundary condition. A polymer chain is modeled by a self-avoiding random walk (SAW) which is generated on the trail of a non-reversal random walk (NRRW) with self-avoiding constraints. The chains are dropped onto the square lattice, one at a time sequentially. If a chain overlaps with previously deposited chains, the attempt is rejected. Once the chain is deposited on the lattice, it sticks on the surface permanently. The deposition rate or equivalently the time scale is fixed with the following algorithm. In unit time $\Delta t=1, L^{2}$ attempts are made to generate and deposit SAW chains each of length $N$ starting from a randomly selected site. As soon as a walk overlaps with the previously deposited site (from other chains or itself), the walk is abandoned, and a new attempt is made to deposit a chain starting from a randomly chosen new site.

Due to the large number of conformational states of polymer chains, the deposition becomes very slow in the late stage. An event-driven method [10,30,31] is, therefore, used to speed up the simulation. This is accomplished by identifying the early part of growing chains, 
i.e., the partial chains and then classifying them periodically as "available" or "not available" for the deposition in an iterative fashion. The subsequent depositions are then made starting from the available partial chains choosing at random. The list of the partial chains is limited by the available computer memory and governs the speed of the program. It appears that there is an optimal list size for a given value of $N$.

Now, in order to maintain the same dynamics, each deposition attempt does not simply advance the time by $\delta t=L^{-2}$ as before, but by an amount $j \delta t$, where $j$ is a random integer with an exponential distribution, $p(1-$ $p)^{j-1} . p$ is the ratio of the number of potentially available partial chains in the list to the total number of chains of partial length $n, L^{2} Z_{n}^{n r r w}$, where $Z_{n}^{n r r w}$ is the number of NRRW chains of length $n$. The random integer can be generated by

$$
j=1+\left\lfloor\frac{\ln \xi}{\ln (1-p)}\right\rfloor,
$$

where $\xi$ is a uniformly distributed random number between 0 and 1.

The simulation is performed on IBM SP2 and fast workstation clusters. The total amount of CPU time for the computation is about six months equivalent of a single DEC AlphaStation 250/266. Variation of the coverage $\theta$ with time $t$ is presented in Fig. 1 1 for various chain lengths. We immediately notice that a rapid increase in the coverage in the short time regime is followed by a very slow growth in the long time. We can divide the characteristic behavior in three time regimes: (1) short time regime, (2) intermediate regime, and (3) very late-stage regime.

The short and very long time regimes (1) and (3) are easily understood. The coverage at very short time is proportional to $t$, since the depositions of nearly all SAWs are accepted. In very late stage [32], the dynamics is controlled by filling independently the last pores. The holes disappear according to $e^{-t / \tau}$, where $1 / \tau$ is the probability that a given hole is being filled (in unit time). For the deposition of chains, the slowest mode is filling the void in exactly two ways (out of $Z_{N}^{n r r w}$ possibilities). Thus

$$
\tau=\frac{Z_{N}^{n r r w}}{2}=2 \cdot 3^{N-2}, \quad N>1
$$

The exponential decays and the prediction for $\tau$ agree very well with simulation data, see Fig. 2 .

In the intermediate time regime $(2), 1<t \ll \tau$, a power-law dependence is observed even on discrete lattice i.e.,

$$
\theta(t) \approx \theta_{J}-c t^{-y},
$$

with an effective exponent $y$. Since the jamming limit $\theta_{J}$ is not known accurately for long chains, it is instructive to consider the derivative of $\theta$ with respect to $t$,

$$
\frac{d \theta}{d t} \propto \frac{1}{t^{1+y}} .
$$

This quantity is plotted in Fig. 3 for various chain lengths. We see a crossover from a power-law variation of the rate of coverage in the intermediate time regime to an exponential decay in the long time especially for short chains. Note that this intermediate regime expands on increasing the chain length. We observe a remarkable power-law behavior over 12 decades in time with the largest chain length $N=30$ for which the true jamming limit is reached in our simulations. Least-square fits in the power-law regime give a reliable estimate of the effective exponent $y$, particularly for $N \leq 30$. Note that the small value of $y \simeq 0.07$ for $N=30$ is stable over a relatively large time scale within the statistical fluctuations (see the inset in Fig. 3, and Fig. 4). A logarithmic fit $\theta(t) \approx \theta_{J}-c / \log (t)$ appears less satisfactory. We see that the magnitude of the exponent $y$ depends systematically on the chain length, $y \simeq 0.50$ for $N=4$ to $y \simeq 0.07$ for $N=30$. To our knowledge, none of the previous studies has shown a size-dependent exponent $y$. A crude extrapolation (see Fig. 4) leads to a small value of $y$ for larger chain length with a possibility for $y \rightarrow 0$ as $N \rightarrow \infty$ within the statistical error.

Let us recall the well-known Swendsen's argument [4] for Feder's law [Eq. (11)] for the adsorption of disks on continuum. At late stage, the pore vanishes according to $e^{-k t}$, where $k \propto l^{d}, l$ is the linear size of the pore, and $d$ is the spatial dimension. Assuming an uniform linear size $l$, the probability distribution for the rate $k$ is $p(k) d k \propto 1 \cdot d l \propto k^{1 / d-1} d k$. Total contribution to the approach to jamming is then

$$
\theta_{J}-\theta(t) \propto \int_{0}^{\infty} k^{\frac{1}{d}-1} e^{-k t} d k \propto t^{-1 / d}
$$

Viot and Tarjus [7 generalized the above result to anisotropic objects and concluded that the approach to jamming is $t^{-1 / d_{f}}$ for monodisperse objects, where $d_{f}$ is the degrees of freedom of the objects. For the objects with regular shape but polydisperse sizes [12], $\theta_{J}-\theta(t) \propto t^{-1 /\left(d_{f}+1\right)}$.

Polymer chains have much more internal degrees of freedom. For sufficiently long chains, the lattice structure becomes less important. The chains can be specified by the orientation of their segments on a coarse-grain level. The number of degrees of freedom is proportional to the number of such segments. Thus, we may identify $d_{f} \propto N$ and consequently $y \propto 1 / N$. In fact the data for $y$ can be roughly characterized by $y \approx 2 / N$.

Alternatively, one may identify the degree of freedom as the number of SAW configurations $Z_{N}^{s a w}$ of length $N$, i.e., $d_{f} \propto Z_{N}^{\text {saw }} \propto N^{\gamma-1} z_{\text {eff }}^{N}$ where $\gamma$ is a critical exponent and $z_{\text {eff }} \leq z-1$, where $z$ is the coordination number [33]. Then, the exponent $y \approx 1 /\left(Z_{N}^{s a w}+1\right)$ becomes much smaller than our estimates. Thus, the application 
of Tarjus and Talbot result [12] is not valid if $d_{f}=Z_{N}^{\text {saw }}$ is assumed. However, in asymptotic limit for large $N$, $y \rightarrow 0$ as $N \rightarrow \infty$ is consistent with the prediction of Tarjus and Talbot.

With the event-driven method we are able to reach jamming much faster which gives us accurate estimates for the jamming coverage $\theta_{J}$, see Table I The jamming coverage decreases with the chain length. It is not clear a priori that the jamming coverage $\theta_{J}(N)$ goes to zero as $N$ approaches infinity. However, assuming,

$$
\theta_{J}(N) \propto N^{-x}
$$

a $\theta_{J}(N)$ versus $N$ plot on a log-log scale (see Fig. 5) leads to a very small exponent $x \approx 0.11$. Note that the magnitude of the exponent $x$ is much smaller than that of ref. [16] $(x \approx 1 / 3)$ which deals with the percolation 34 ] of chains. The jamming coverage in the percolation, i.e., the maximum value of the volume fraction is the "first jamming coverage" which is far from the asymptotic limit of our "true jamming coverage" here.

We have also analyzed the conformation of the chains during the deposition process. For SAW, it is well-known [28,29 that the radius of gyration or end-to-end distance varies with chain length as

$$
R_{g} \propto R_{e} \propto N^{3 / 4}
$$

The question now is whether this law is modified for the chains deposited on the lattice. Our numerical data indicate that the average radius of gyration is less sensitive to packing. One of the possible reasons for not observing much change in the power-law dependence from that of a free SAW, is the fact that the conformations of the chains in their early stage of deposition dominate the average radius of gyration. However, due to the irreversible nature of the problem, the chains have memory of their arrival time in the sense that their conformation and correlation depend on $t$. In fact, we do observe that the radius of gyration decreases systematically with time especially in the long time regime.

We thank Gan Chee Kwan, and Grace M Foo for useful discussions. This work is supported in part by the Faculty Research Grant RP950601. Part of the computation was performed on IBM SP2 of the National Supercomputing Research Centre. Warm hospitality during his sabbatical visit at the NUS is acknowledged by RBP.

* Permanent address: The Program in Scientific Computing, Department of Physics and Astronomy, University of Southern Mississippi, Hattiesburg, MS 39406, USA.

[1] For a review, see: J. W. Evans, Rev. Mod. Phys. 65, 1281 (1993).
[2] J. Feder, J. Theor. Biol. 87, 237 (1980).

[3] Y. Pomeau, J. Phys. A 13, L193 (1980).

[4] R. H. Swendsen, Phys. Rev. A 24, 504 (1981).

[5] P. Schaaf and J. Talbot, Phys. Rev. Lett. 62, 175 (1989); J. Talbot, G. Tarjus, and P. Schaaf, Phys. Rev. A 40, 4808 (1989).

[6] R. D. Vigil and R. M. Ziff, J. Chem. Phys. 91, 2599 (1989).

[7] P. Viot and G. Tarjus, Europhys. Lett. 13, 295 (1990).

[8] R. Dickman, J.-S. Wang, and I. Jensen, J. Chem. Phys. 94, 8252 (1991).

[9] N. M. Švrakić and M. Henkel, J. Phys. (Paris) I1, 791 (1991).

[10] B. J. Brosilow, R. M. Ziff, and R. D. Vigil, Phys. Rev. A 43, 631 (1991).

[11] G. Tarjus and P. Viot, Phys. Rev. Lett. 67, 1875 (1991).

[12] G. Tarjus and J. Talbot, J. Phys. A 24, L913 (1991).

[13] P. Meakin and R. Jullien, Phys. Rev. A 46, 2029 (1992); Physica A 187, 475 (1992).

[14] P. Viot, G. Tarjus, S. M. Ricci, and J. Talbot, J. Chem. Phys. 97, 5212 (1992); S. M. Ricci, J. Talbot, G. Tarjus, and P. Viot, J. Chem. Phys. 97, 5219 (1992).

[15] V. Privman, J.-S. Wang, and P. Nielaba, Phys. Rev. B 43, 3366 (1990); J.-S. Wang, V. Privman, and P. Nielaba, Mod. Phys. Lett. B7, 189 (1993); J.-S. Wang, P. Nielaba, and V. Privman, Physica A 199, 527 (1993).

[16] J. L. Becklehimer and R. B. Pandey, J. Stat. Phys. 75, 765 (1994).

[17] R. S. Sinkovits and R. B. Pandey, J. Stat. Phys. 74, 457 (1994).

[18] A. Baram and M. Fixman, J. Chem. Phys. 103, 1929 (1995).

[19] P. J. Flory, J. Am. Chem. Soc. 61, 1518 (1939).

[20] L. Finegold and J. T. Donnell, Nature 278, 443 (1979).

[21] N. O. Wolf, D. R. Burgess, and D. K. Hoffman, Surf. Sci. 100, 453 (1980).

[22] G. Y. Onoda and E. G. Liniger, Phys. Rev. A 33, 715 (1986).

[23] V. Privman, H. L. Frisch, N. Ryde, and E. Matijević, J. Chem. Soc. Faraday Trans. 87, 1371 (1991).

[24] J. J. Ramsden, Phys. Rev. Lett. 71, 295 (1993); J. J. Ramsden, G. I. Bachmanova, and A. I. Archakov, Phys. Rev. E 50, 5072 (1994).

[25] A. Rényi, Publ. Math. Inst. Hung. Acad. Sci. 3, 109 (1958).

[26] J. J. Gonzalez, P. C. Hemmer, and J. S. Høye, Chem. Phys. 3, 228 (1974).

[27] M. C. Bartelt and V. Privman, Int. J. Mod. Phys. B 5, 2882 (1991).

[28] P. J. Flory, Statistical Mechanics of Chain Molecules (Interscience, New York, 1969).

[29] P. G. de Gennes, Scaling Concepts in Polymer Physics (Cornell University Press, Ithaca, NY, 1979).

[30] J.-S. Wang, Inter. J. Mod. Phys. C 5, 707 (1994).

[31] A. B. Bortz, M. H. Kalos, and J. L. Lebowitz, J. Comp. Phys. 17, 10 (1975).

[32] M. C. Bartelt and V. Privman, Phys. Rev. A44, R2227 (1991).

[33] K. Binder and D. W. Heermann, Monte Carlo Simulation in Statistical Physics, p. 10, (Springer-Verlag, Berlin, 1988). 
[34] D. Stauffer and A. Aharony, Introduction to Percolation, 2nd Ed., (Taylor \& Francis, London, 1994).

FIG. 1. Coverage as a function of time, for chain length $N$ indicated by the number. See table for the statistics on the sample size and the number of independent runs.

FIG. 2. Semi-logarithmic plot for the difference between jamming coverage and coverage at time $t$, for chain lengths $N=2,5,10$, and 15 . For a better view of all the curves on the same plot, we plotted again the normalized time $t / \tau$. The straight line is the pure exponential decay $e^{-t}$ for $N=1$. The insert shows $\tau$ as a function of $N$, the straight line is the theoretical prediction, Eq. (3).

FIG. 3. Derivative of coverage vs. time on a log-log scale. The number indicates chain length $N$. Insert shows the variation of exponent $y$ with time evaluated at a regular interval for $N=30$. The statistics is presented in table 1 .

FIG. 4. Exponent $y$ obtained by least-squares fits of Fig. 3, plotted against inverse chain length.

FIG. 5. Jamming coverage versus chain length on a logarithmic scale.

TABLE I. Jamming coverage $\theta_{J}=\theta(t \rightarrow \infty)$ for various chain lengths. Statistical errors on the last digits are indicated by the numbers in the parentheses.

\begin{tabular}{rlrr}
\hline \hline$N$ & $\theta_{J}$ (error) & $L$ & number of runs \\
\hline 1 & 1 & & \\
2 & $0.906820(2)$ & 1000 & 26200 \\
3 & $0.858296(4)$ & 500 & 34000 \\
4 & $0.837055(13)$ & 500 & 14000 \\
5 & $0.81235(1)$ & 500 & 7501 \\
7 & $0.78558(2)$ & 500 & 2320 \\
10 & $0.75895(1)$ & 500 & 4701 \\
15 & $0.72473(8)$ & 500 & 700 \\
20 & $0.70178(10)$ & 500 & 33 \\
30 & $0.6683(4)$ & 500 & 28 \\
40 & $0.654(9)$ & 200 & 7 \\
\hline \hline
\end{tabular}



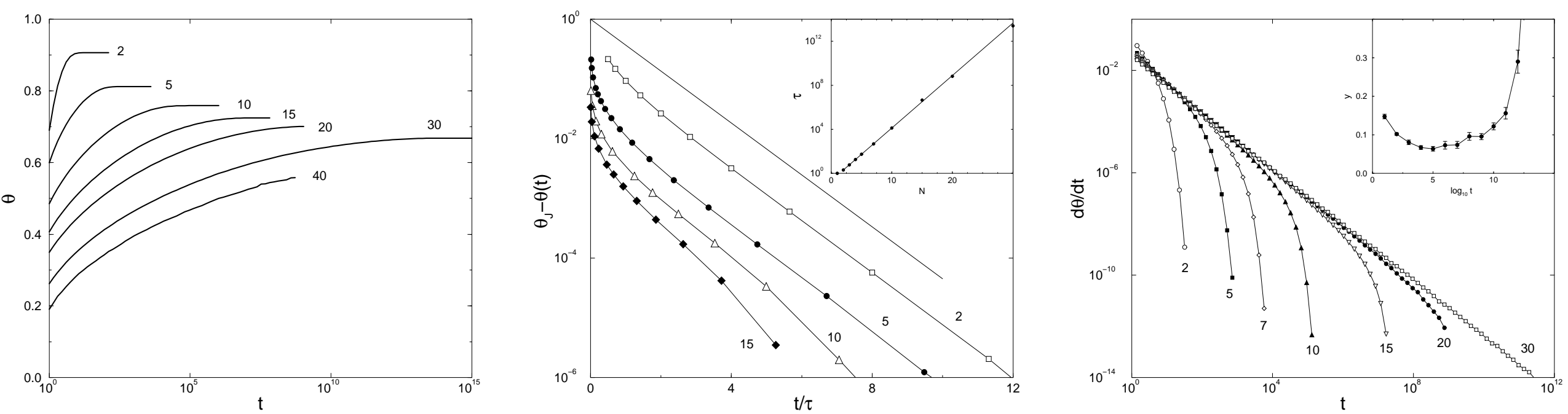

Fig. 4
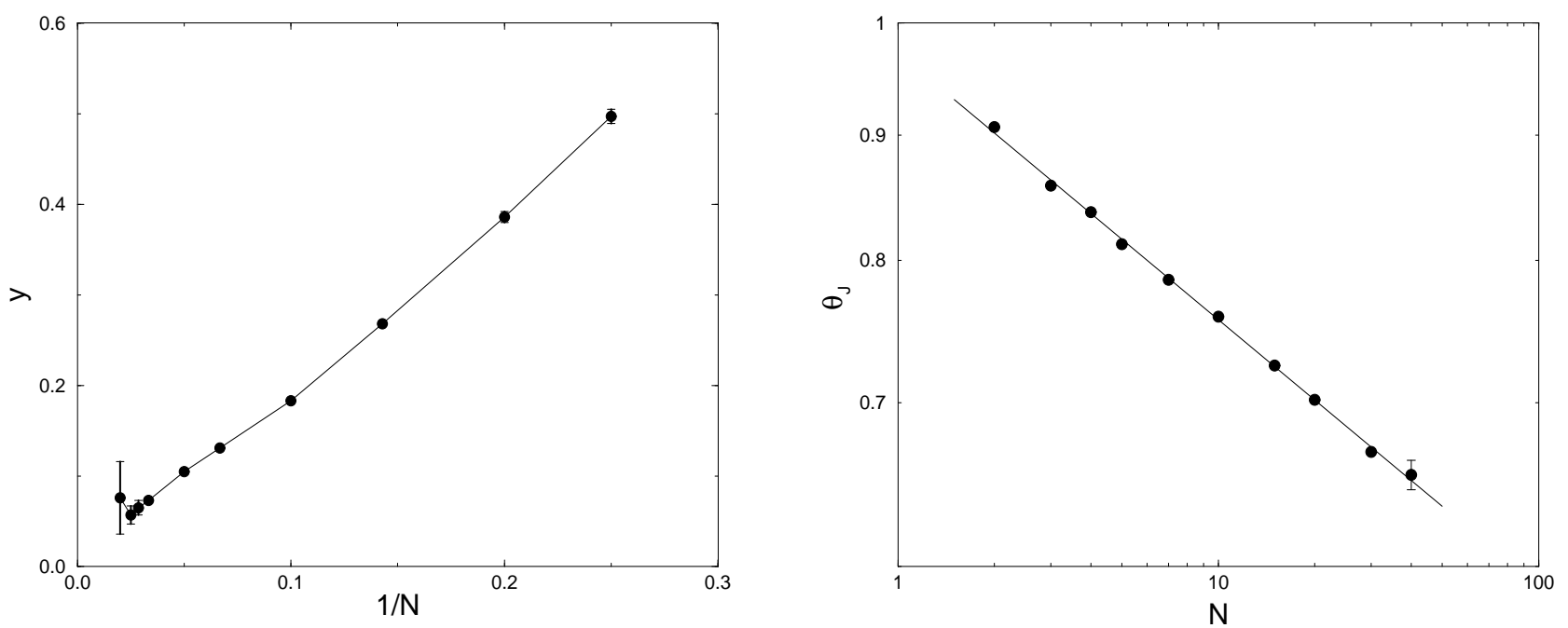